\title{
Infrared Period-Luminosity Relations of RR Lyraes in M5 and M15
}

\author{
T. Liu ${ }^{1}$, K. A. Janes ${ }^{2}$ \\ ${ }^{1}$ University of California at Los Angeles, ${ }^{2}$ Boston University
}

\begin{abstract}
We have carried out $K$ band photometric observations of RR Lyrae stars in two globular clusters which both have large populations of RR Lyraes but different characteristics: the moderately metal-rich cluster $\mathrm{M} 5([\mathrm{Fe} / \mathrm{H}]=-1.40)$ and the metal-poor one $\mathrm{M} 15([\mathrm{Fe} / \mathrm{H}]=-2.15)$. The purpose is to accurately calibrate the linear relationship between $\mathrm{RR}$ Lyrae infrared $(\mathrm{K})$ absolute magnitudes and their periods that has been confirmed by recent Baade-Wesselink type studies of RR Lyraes and IR photometry of cluster variables. A total of 47 RR Lyraes in M15 was observed and each has more than 8 measures on the average, which allows the accurate determination of a mean $K$ magnitude for each star. In M5 44 stars have been observed with each RR Lyrae having 4 measurements. Our preliminary results show that the RR Lyrae infrared period-luminosity relations for the two clusters have roughly the same slope, despite the fact that they have a large metallicity difference. This suggests that the metallicity effect on the $\left\langle M_{K}\right\rangle-\log P$ relation is indeed small as one would expect. The M5 and M15 RR Lyrae IR photometry gives a reliable determination for the slope of the infrared period-luminosity relation because of the large number of stars measured. A well-calibrated $\left\langle M_{K}\right\rangle-\log P$ relation will be very useful in distance determinations to heavily reddened star regions such as the Galactic center and globular clusters in the Galactic bulge.
\end{abstract}
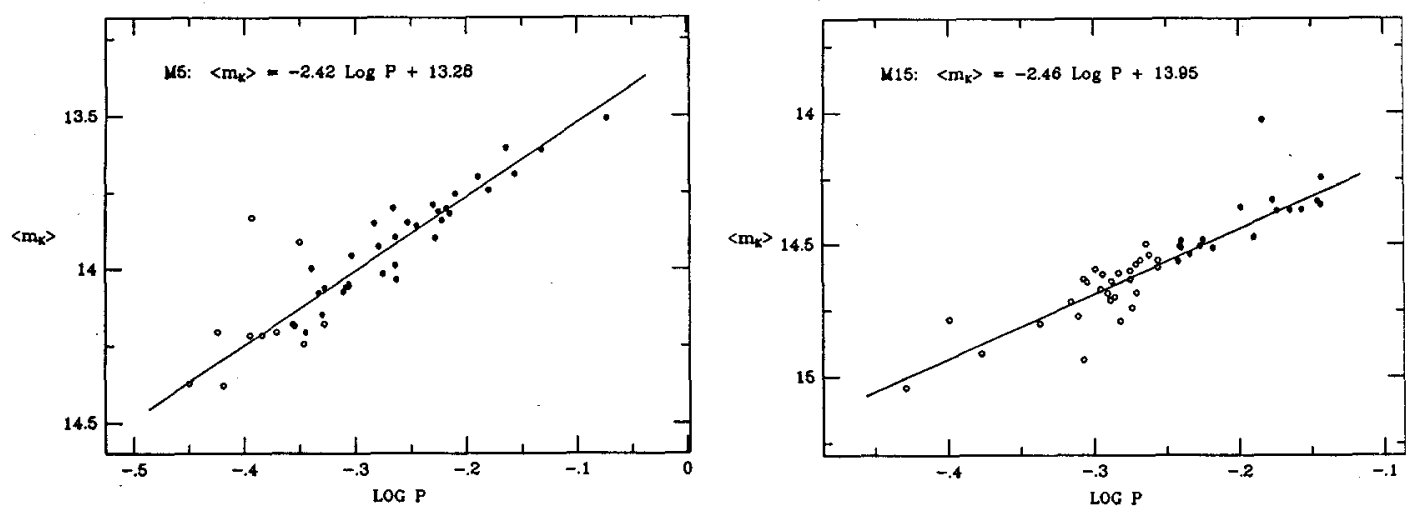

Figure $1\left\langle m_{K}\right\rangle$ vs. $\log P$ for RR Lyrae stars in M5 and M15. Filled circles are ab-type variables, while open circles represent c-type stars. The solid lines are linear least-squares fit to the data. 